\title{
AIR ASSISTANCE TO PILOTS, AN AID IN SITUATION OF EMERGENCY LANDING OF AIRPLANES
}

\author{
Jeena Shone ${ }^{1}$ \\ ${ }^{I}$ Assistant Professor, Electronics and Communication, T.John Institute of Technology, Karnataka, India
}

\begin{abstract}
Air transportation has become one of the key transportation system in today's world. Especially when business and trade across the world is increasing. This then brings the necessity to make the aero plane journeys safer because of the increase in number of people utilizing its possibility. Airplane paths are designed in such a way that they face least obstruction in its path and experience less turbulence. However, sometimes unexpected conditions come up like an engine failure, extreme weather conditions, shortage of fuel etc... where an emergency landing become necessary. For this purpose, many automated systems are designed. This paper gives the reader an overview of some of these systems and an insight to an image processing system for the same and its performance comparison when two types of clustering is used.
\end{abstract}

Keywords: clustering, fuzzy c- means, canny edge detector etc...

\section{INTRODUCTION}

Aircraft transport has become one among the most used transportation facilities in today's modern world. Comfort and safety has been the two most important criterion that is taken care of. Safety of the passengers is a key factor as there will be many passengers on board. As in the early days of flight operations, the pilot was solely responsible for the conduct of a flight. At the time of emergency landing the pilot had to look through his cockpit and make decisions to choose a suitable land for emergency landing, where in he may or may not succeed. Later came in many technological advancement which paved way for many image processing systems to reduce the risks in situation of emergency landing. High resolution cameras were fitted in the flight that takes images of ground and using image processing systems, the image is processed and a suitable site is being shown to the pilot which he can verify and make an appropriate decision[1].

\section{RECENT ADVANCEMENT}

The first system used for the purpose was composed of a RADAR equipment, which using the radar signal will identify the obstructions on the surface and will give the pilot information about the obstructions. Next, when Laser was introduced, LIDAR systems were used which would use laser beams to detect obstructions[4]. However all these systems used could only tell the pilot about the presence of the obstruction, but the pilot will not be able to see the actual visuals. Then when image processing systems gained its significance, sophisticated systems were designed for the purpose. It would take photographs of the nearby area and process the image making use of pixel values and find the safer regions[1]. Options will be given to the pilot with images so that he can visualize it and make decisions accordingly.

\section{ARCHITECTURE}

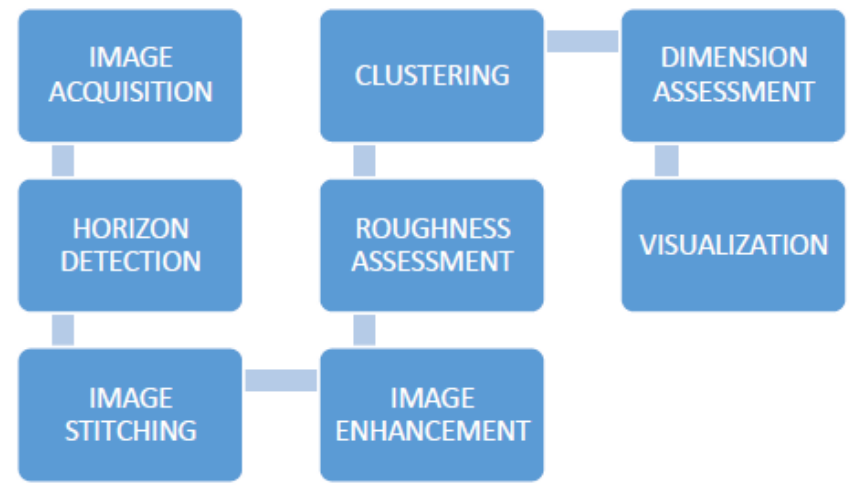

Fig -1: Basic architecture

In reference to the system proposed by [1], the architecture consists of the pre-processing stage (image acquisition, image stitching, image enhancement) and the processing stage (horizon detection, roughness assessment, classification and segmentation and dimension assessment).

\subsection{Pre-Processing Stage}

Images are obtained using high resolution cameras fitted on to the aero-plane mast at the front. Fig:2 shows a sample image which has to be processed. [1].The obtained images will be stitched together to give a wide range image for processing. The stitched image may not be clear or may be unsuitable for further processing due to weather conditions. So it is fine tuned using a filter or any other de-haze operation. Nowadays water proof cameras and high clarity cameras are used to tackle the weather conditions that may arise.

\subsection{Processing Stage}

The pre-processed image now must undergo various steps in its processing stage in order to get the desired output result. 
The first step is to determine the horizon of the image. It is understood that the images that the camera captures will have both sky and land and we need to process only the land portion of the image. Pixel values of sky will be almost a constant so it can be differentiated easily from the land. Hough transform is utilized here for the same. Fig:4 gives horizon detected image.[1]

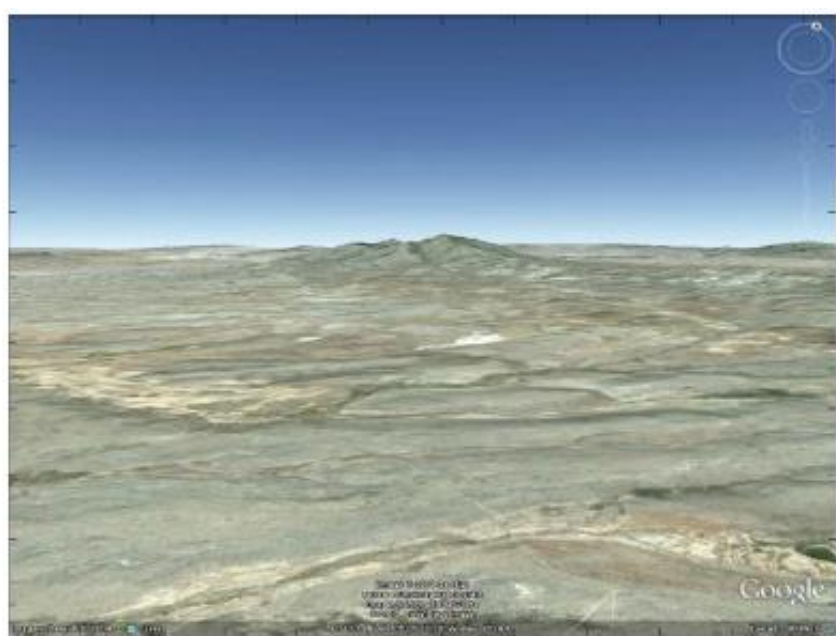

Fig -2:Sample picture

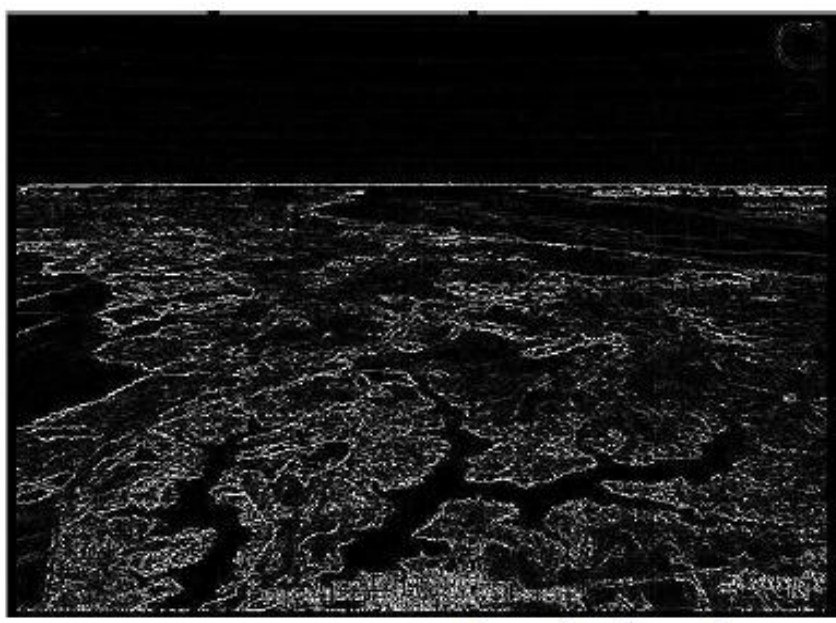

Fig -3: Image after edge detection

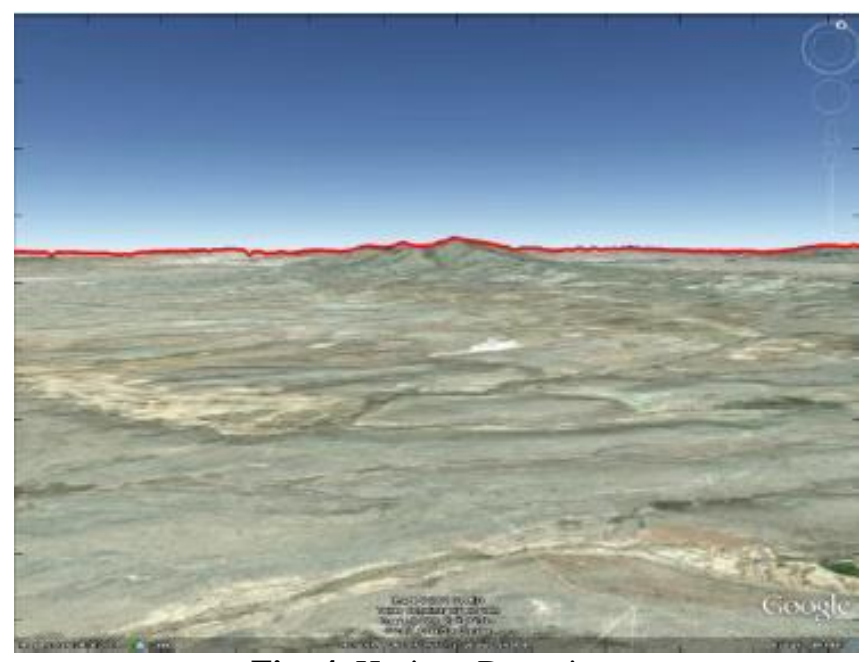

Fig -4: Horizon Detection
After the horizon is detected, roughness assessment is done to distinguish the hard surface from the soft surface. Which is done using a hazard determination technique for which the pixel values are considered. Canny edge detector is used to determine the edge strength. Result for canny edge detection is given in Fig:3. [2]

$$
\begin{aligned}
\mathrm{CHS}_{B} & =\sum_{p \in B} H\left(\mathrm{ES}_{p}\right) \\
H\left(\mathrm{ES}_{p}\right) & = \begin{cases}1 & \mathrm{ES}_{p}>T \\
0 & \mathrm{ES}_{p} \leq T\end{cases}
\end{aligned}
$$

Were, ES is the edge strength of each pixel $\mathrm{P}$ and $\mathrm{H}()$ is the hazard indication factor. $\mathrm{T}$ is a threshold set.[1]

Classification of the regions are done are done using clustering technique. Normal clustering is tried first and then repeated with k-mean clustering and fuzzy c-mean clustering. It is evident from the result that Fuzzy c-means clustering is better compared to the first two.[2]

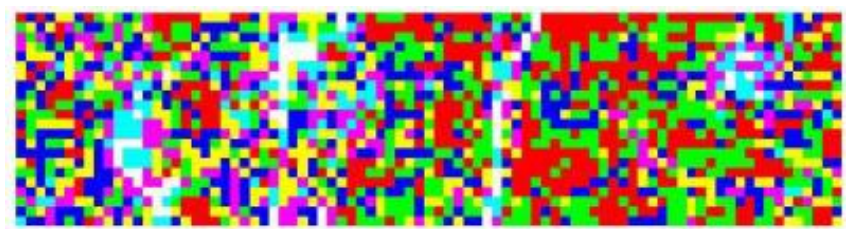

Fig -4: clustered image

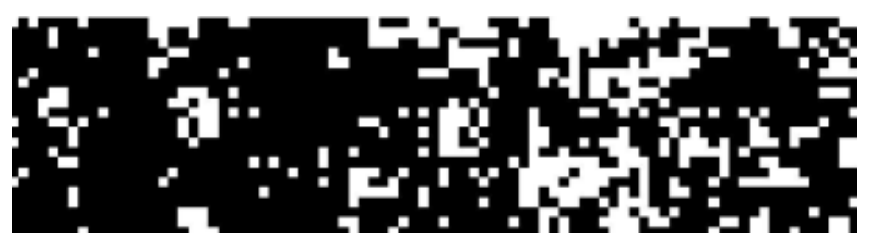

Fig -5: Smooth areas identified

After clustering of the image is completed, all the rough areas are removed and only the softer regions are taken for further processing as shown in Fig:5 [2]. Now the dimensions has to be obtained.. The area requirement of different flights for landing will not be the same. For example, a helicopter requires lesser area compared to passenger flight. Accordingly the pilot chooses the best available options from the obtained choices which the system gives.[2]

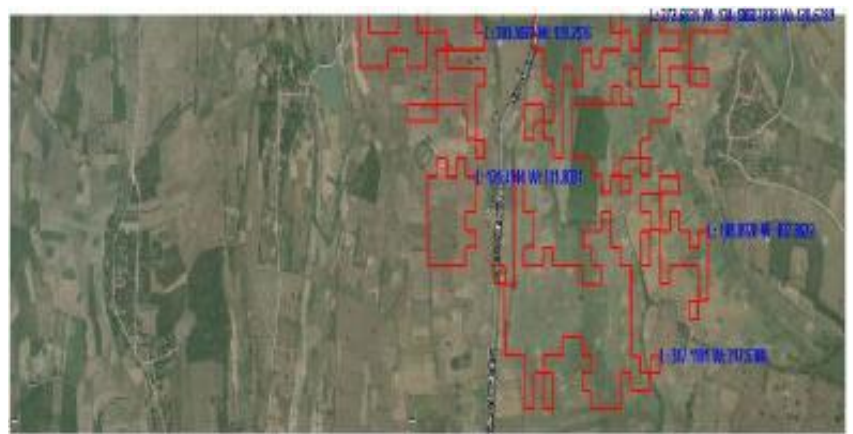

Fig -6: Dimension Assessment 


\section{RESULT}

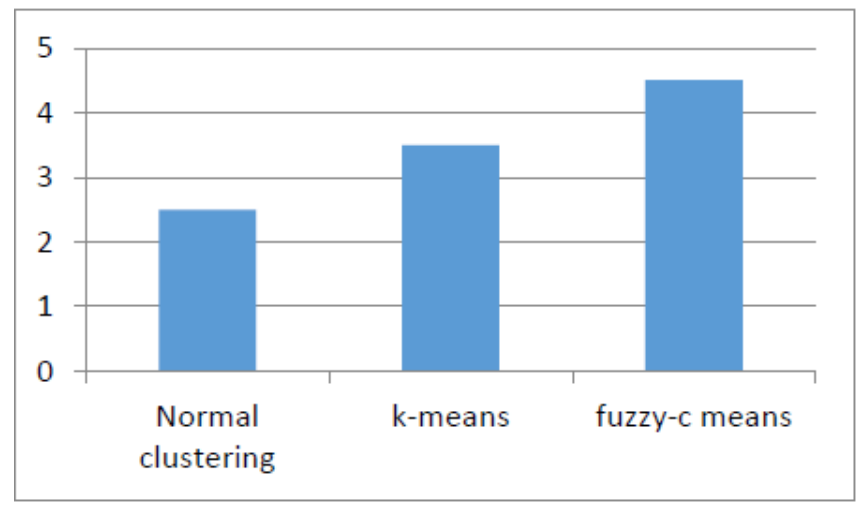

The output comparison of the different clustering is shown in a bar graph here. It is evident from the graph that fuzzy cmean clustering is more efficient that the other types of clustering.

\section{CONCLUSION}

This paper presents an automatic safe landing system for aircrafts in case of emergency landing. The proposed system will eliminate the limitation of human eyes and helps pilot to find the safe landing site and more importantly saves time for the pilot to devote for necessary actions because the flight may constantly loose altitude at the time of emergency. So time plays a crucial role. Here, normal clustering, fuzzy-c means and k-means clustering is done and compared. And it is seen that fuzzy-c means clustering is better than the existing technique as proposed by [1]. However it has got certain limitations as there is requirement of more resources and time for computations. To meet the practical requirements, a threshold considering the terrain conditions can be set for a particular region. Also LIDAR systems can be used along with the proposed system to obtain higher accuracy on hazard level information of the surface.

\section{ACKNOWLEDGEMENT}

This paper work would not have been possible without the support and guidance of Mr. Arun, who helped me throughout the project development. Also my HOD, Dr. Arun Vikas Singh who motivated me to do this paper.

\section{REFERENCES}

[1]. Yu-Fei Shen,Old Dominion University,Zia-Ur Rahman, Senior Member, IEEE Nasa Langley Research Center, Dean Krusienski, Member, IEEE,,Jiang Li, Member, IEEE .”A Vision-Based Automatic Safe, Landing-Site Detection System", IEEE Transactions On Aerospace And Electronic Systems Vol. 49, No. 1 January 2013

[2]. Pilot Assistive Safe Landing Site Detection System, an Experimentation Using Fuzzy C Mean Clustering Jeena Wilson, IJMER, Vol. 4 | Iss.10| Oct. 2014 | 32|

[3]. Todd Templeton, David Hyunchul Shim, Christopher Geyer, and S. Shankar Sastry. "Autonomous Vision-based Landing and Terrain Mapping Using an MPC-controlled
Unmanned Rotorcraft", Dept. of Aerospace Engineering, Korea Advanced Institute of Science and Technology,2007. [4]. Srikanth Saripalli, Student Member, IEEE, James F. Montgomery, And Gaurav S. Sukhatme, Member, IEEE."Visually-Guided Landing Of An Unmanned Aerial Vehicle".IEEE Transaction On Robotics And Automation, Vol 19, No 3,June 2013

[5]. Paul Williams, Michael Crump BAE Systems Australia.'Intelligent Landing System For Landing Uavs At Unsurveyed Airfields", 28th International Congress Of The Aeronautical Sciences

[6]. Daniel Fitzgerald 1, Dr Rodney Walker 1, Dr Duncan Campbell."A Vision Based Emergency Forced Landing System for an Autonomous UAV', In Proceedings Australian InternationalAer ospace Congress Conference, Melbourne Australia, Melbourne,Australia,2005.

[7]. Scott R. Ploen Homayoun Seraji Charles E. Kinney,'Determination of Spacecraft Landing Footprint for Safe Planetary Landing", IEEE Transactions On Aerospace And Electronic Systems Vol. 45, No. 1 January 2009.

\section{BIOGRAPHY}

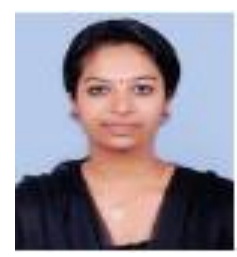

Jeena Shone is currently working as an Assistant Professor in T.John Institute Of Technology, Bangalore. She completed her M.Tech from Federal Institute of Science and Technology, Ernakulam, Kerala. 